\title{
Design of Monitoring Platform of Hydraulic Support Operation Pressure Based on ARM and CAN Bus
}

\author{
Wei $\mathrm{LI}^{1, a}$, Cheng DING ${ }^{2, b}$ \\ ${ }^{1}$ School of Mechanical Engineering, Anhui University of Science\&Technology, Huainan, \\ AnHui,China \\ ${ }^{2}$ Hefei Design Research Institute for Coal Industry, Hefei, Anhui,China \\ aliwei7511@126.com, bdingchengyouxiang@qq.com \\ * Corresponding author. Tel:+86-554-6668934
}

Keywords: hydraulic support; operation pressure; monitoring platform; CAN bus

Abstract. According to the shortages existed in current methods of monitoring on hydraulic support operation pressure, this paper developed a new monitoring system in which monitoring platform with multi-layer designed based on embedded system and CAN bus technology was introduced. The platform has integrated multi-function together, such as real-time monitoring, historical queries and communication in networks, to realize an intelligent monitoring on operation pressure by networks.

\section{Introduction}

Hydraulic supports are the main support equipment used in fully-mechanized coal face, of which operation pressure,due to the relation with ground pressure, is necessary for coal mine safety monitoring system.Traditional method of getting hydraulic supports operation pressure,that collecting pressure data periodically by worker from the self-recorder equipped in each hydraulic support and sending the data to ground control hub, is now no longer meet the demand of remote and dynamic monitoring system in modern coal mine. Except for the electro-hydraulic controlled support that could be detected real-timely, most hydraulic supports controlled by manual valves thus need to be equipped with operation pressure monitoring system.

However, some problems have been found when the monitoring systems were applied, such as complex structure of hardware, redundant function, limited ability in data procession by RS-485 BUS, as well as heavy explosion-proof shell with bad cooling effect.This paper thus developed a new remote monitoring system of hydraulic support operation pressure based on ARM and CAN bus, with a compact structure and ability of dealing with mass data.

\section{Overall structure of system}

The overall structure of the system was composed of pressure monitoring sub-stations, monitoring platform and network server, as shown in figure 1.

The sub-stations, including of pressure transducers and data acquisition card, played the role of measuring the operation pressure of each hydraulic support and sending the data to the upper monitoring platform by CAN bus. The platform connected low networks of CAN bus with top Ethernet ring networks, which could receive and process the data from the sub-station and give alarm at the case of overrun pressure, as well as package all the data received and send it to network switch through Ethernet interfaces. The server consist of network server, host computer and corresponding software. By matching IP address, the server could arrange and save different received message of coal face position, of hydraulic support operation pressure and of alarm, and provide the results for staff in control center to realize a real-time supervision and daily management.

According to overall structure of the system, when applied in coal face, each pressure monitoring sub-station should be installed under the top beam of hydraulic support, and the pressure transducer was connected with the hydraulic oil chamber of props to obtain the value of pressure. All the 
sub-stations were connected one by one by cable to realize power provision and communication. The monitoring platform installed in cross-heading was connected with the nearest sub-station in order to collect and process the data from all the sub-stations by CAN bus. The data then was send to Ethernet switch through the use of TCP/IP protocol, so people in control centers of coal mine and of senior group could make remote and dynamic management on ground.

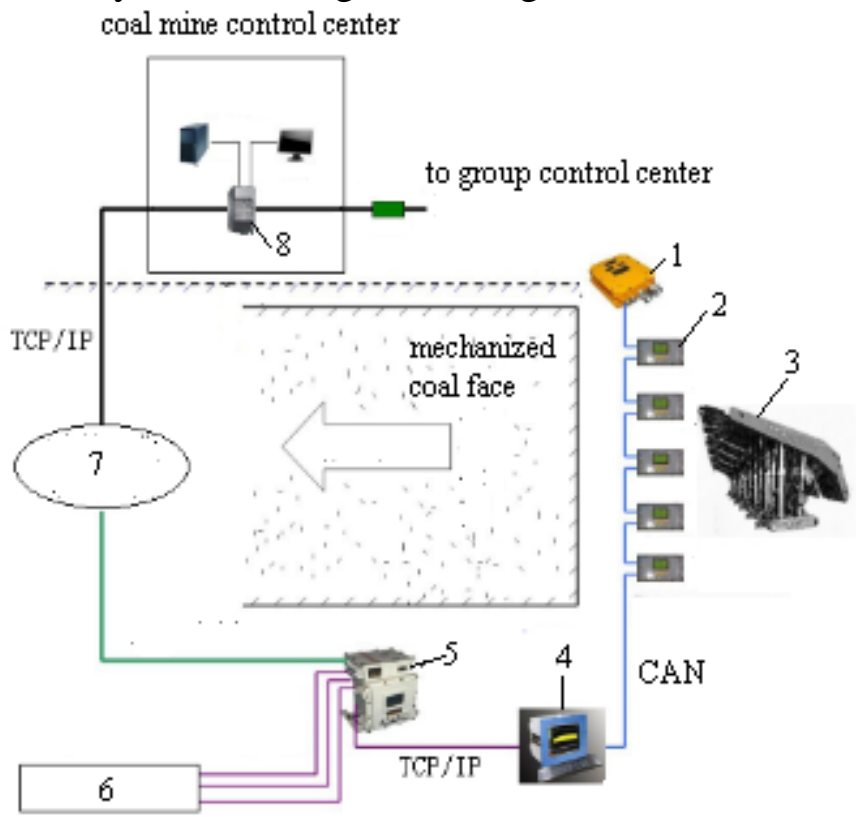

1. flameproof power 2. pressure monitoring sub-station 3. hydraulic support 4. monitoring platform

5. Ethernet switch 6. other monitoring system 7. industrial Ethernet-ring 8. network sever

Fig.1 Structure of hydraulic support pressure monitoring system

\section{Hardware design of monitoring platform}

According to the function of the platform, its hardware was designed as shown in figure 2. Inside the hardware, a chip of ARM (STM32F107VCT6) was chosen as microprocessor, whose minimum system include power circuit, clock circuit and reset circuit. In addition, JTAG debugging circuit, double CAN interfaces circuit, 10M/100M Ethernet interface circuit and module of touch screen, were also designed.

\section{Design of CAN bus module}

CAN bus is an useful field bus technology in distributed control and real-time control system with serial communication. Because CAN bus interface was integrated within STM32F107VCT6, another transceiver chip of SN65HVD230 was chosen due to its advantages of low power consumption, of good reliability and immunity to electromagnetic disturbance.

The circuit of CAN bus module was shown in figure 3. In the circuit, output pin Tx of CAN controller was connected with input pin TXD of SN65HVD230 to send data into CAN network, while input pin Rx was connect with output pin RXD to receive data. A slope resistor $(0 \sim 100 \mathrm{k} \Omega)$ was connected with mode select port Rs to realize three operation mode switch.

\section{Ethernet communication module}

Ethernet communication module was also integrated in STM32F107VCT6, meeting MAC Ethernet protocol of IEEE-802.3 standard, AMBA2.0 standard and RMII standard. The module could be adjusted flexibly for different application, and include a site interface of SMI to communicate with outsider by PHY.

\section{Electromagnetic anti-interference design}

As for the monitoring platform was used in complex electromagnet environment underground, anti-interference measures has been applied in design of overall structure and PCB circuit, as 
concluded in the following: Metal cabinet was used as the shell of the monitoring platform in order to shield it from electromagnetic filed. In connection the platform with the sub-station, cables and connector were unified. Four-layer board was applied in PCB circuit, in which the first and the fourth layer was signal layer, and the second was earthing and the third was power layer. Such layout had several advantages, including large wiring area, isolation signal from disturbance, reduction of characteristic resistance between the signal layer and power return path.

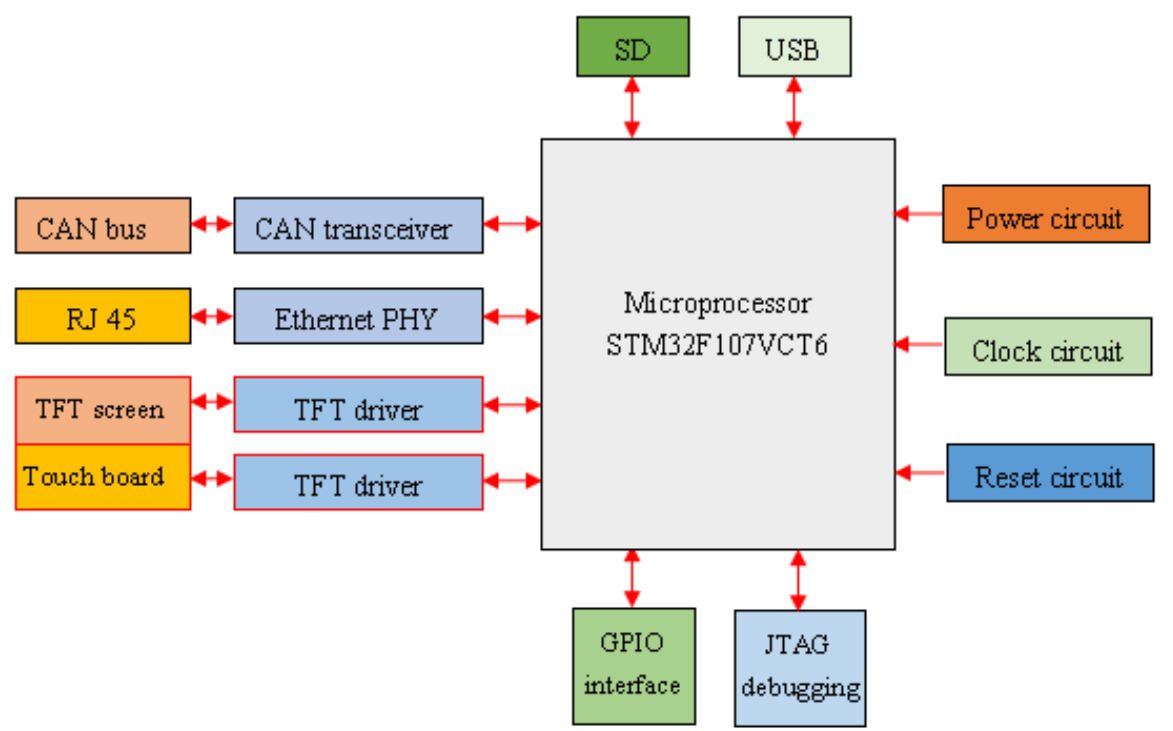

Fig. 2 Hardware structure of monitoring platform

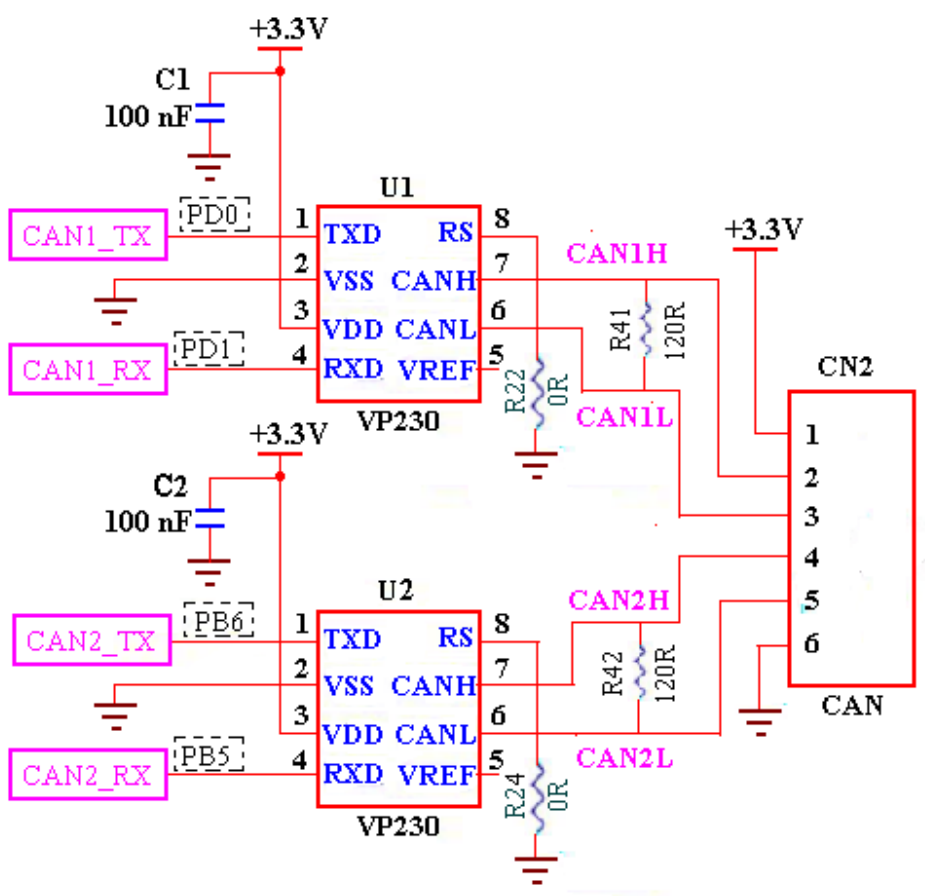

Fig. 3 Circuit of CAN bus module interface

\section{Software design of monitoring platform}

\section{Operation system and development environment}

According the hardware, $\mu \mathrm{C} / \mathrm{OS}$-II, one of the popular operation system for embedded system, as well as was integrated development environment of RealView MDK, was chosen to design software. Because $\mu \mathrm{C} / \mathrm{OS}$-II itself could not support peripheral units, such as document system, stack protocol and graphical interface, so the source code of $\mu \mathrm{C} / \mathrm{OS}$-II system was expanded before an embedded operation system with real-time multitask was developed. 


\section{Tasks division of monitoring platform}

Modules of the platform should be coordinated to realize an efficient real-time monitoring on operation pressure of hydraulic supports in the whole mechanized coal face. So, tasks of the platform were classified as 7 tasks, as shown in figure 4.Tasks 1-7 meant respectively as initialization task, communication through CAN bus, data display, process of touching keys, interfaces switch, sending data through Ethernet and process of LwIP network protocol stack.

\section{Software design of main function}

In order to operate $\mu \mathrm{C} / \mathrm{OS}$ system, several functions, including OSInit( ), OSTaskStart( ), OS TimeSet( ) and OS Start( ) should be called in turn. These functions consisted of main function which carried out according to the flow shown in figure 5.

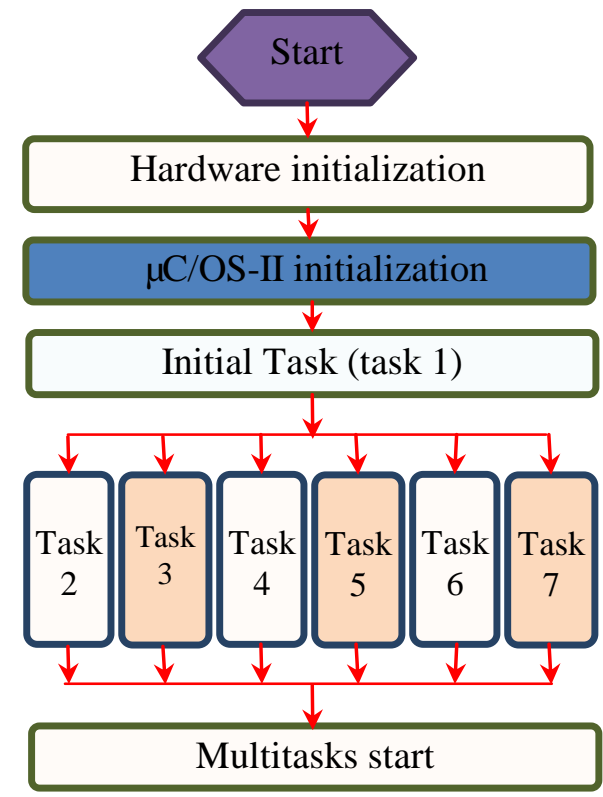

Fig.4 Classify of multitasks

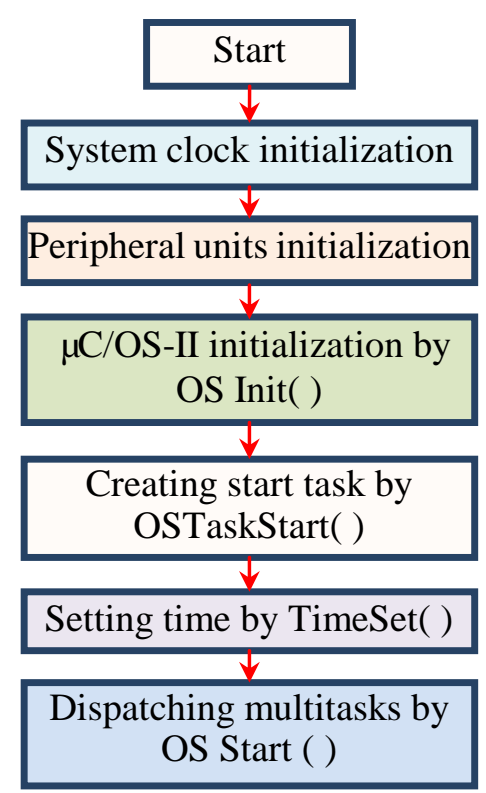

Fig.5 Flow of main function

\section{Debugging of communication module}

An CAN bus transceiver with USB interface was applied to debug data communication. When parameter was set correctly, the platform could receive the data send by the transceiver according to CAN bus protocol and display the data on screen. Figure 6 showed that data communication through CAN bus was succeed.

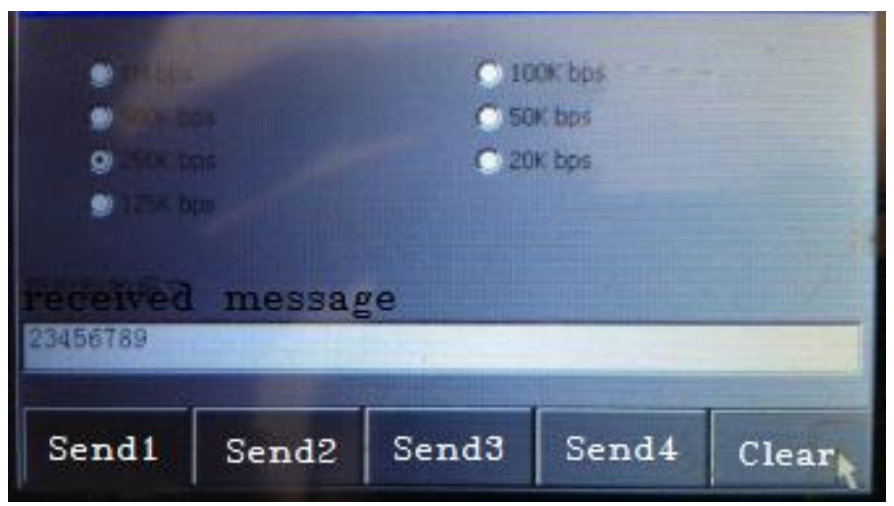

Fig.6 Debugging result of data communication

\section{Conclusion}

The monitoring platform of hydraulic support operation pressure, designed based on ARM and CAN bus technology, has integrated multi-function together, such as real-time monitoring, historical 
queries and communication in networks, to realize an intelligent monitoring on operation pressure by networks. It could be applied in safety monitoring system of coal mine due to its advantages of high precision, operation stability and low cost.

\section{References}

[1] SUN Ji-ping. Research of Key Technologies for Mine Safety and Efficiency Monitoring (In Chinese)[J]. Industry and Mine Automation/Gong Kuang Zi Dong Hua, 2012, (12):1-5

[2] LIU Ying, LIANG Yu, ZHANG Xiaoguang. Design of A Monitoring System for Hydraulic Supports Based on ARM (In Chinese)[J]. /Kuang Shan Ji Xie. 2010, 38(11):6-10

[3] Wang Kai. Design of Pressure Monitoring System for Hydraulic Support Based on LabVIEW(In Chinese)[J]. Colliery Mechanical\&Electrical Technology/Mei Kuang Ji Dian, 2015,(2):71-74

[4] DING En-jie, MENG Xiang, LI Xiao. etc. Design of Coal Mine Hydraulic Support Pressure Monitoring System Based on Wireless Sensor Networks(In Chinese)[J]. Coal Mine Machinery/Mei Kuang Ji Xie, 2010, 31(10):139-141

[5] DING Cheng, MENG Guo-ying, CUI Guo-liang, etc. Design of The Monitoring System Platform of the Hydraulic Support Based on the ARM and CAN BUS (In Chinese)[J]. Instrument Technique and Sensor/Yi Biao Ji Shu Yu Chuan Gan Qi, 2012,(11):169-171 